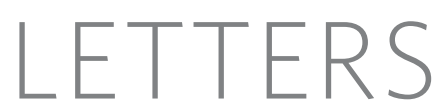

\title{
Coordinated regulation of Arabidopsis thaliana development by light and gibberellins
}

\author{
Suhua Feng ${ }^{1,2} \uparrow$, Cristina Martinez ${ }^{1 *}$, Giuliana Gusmaroli ${ }^{1 *}$, Yu Wang ${ }^{3 *}$, Junli Zhou ${ }^{2 *}$, Feng Wang ${ }^{2}$, Liying Chen $^{2}$, \\ Lu Yu ${ }^{2}$, Juan M. Iglesias-Pedraz ${ }^{4}$, Stefan Kircher ${ }^{5}$, Eberhard Schäfer ${ }^{5}$, Xiangdong Fu ${ }^{6}$, Liu-Min Fan ${ }^{3}$ \\ \& Xing Wang Deng ${ }^{1,2,3}$
}

Light and gibberellins (GAs) mediate many essential and partially overlapping plant developmental processes. DELLA proteins are GA-signalling repressors that block GA-induced development ${ }^{1}$. GA induces degradation of DELLA proteins via the ubiquitin/ proteasome pathway ${ }^{2}$, but light promotes accumulation of DELLA proteins by reducing GA levels ${ }^{3}$. It was proposed that DELLA proteins restrain plant growth largely through their effect on gene expression ${ }^{4,5}$. However, the precise mechanism of their function in coordinating GA signalling and gene expression remains unknown. Here we characterize a nuclear protein interaction cascade mediating transduction of GA signals to the activity regulation of a light-responsive transcription factor. In the absence of GA, nuclear-localized DELLA proteins accumulate to higher levels, interact with phytochrome-interacting factor 3 (PIF3, a bHLH-type transcription factor) and prevent PIF3 from binding to its target gene promoters and regulating gene expression, and therefore abrogate PIF3-mediated light control of hypocotyl elongation. In the presence of GA, GID1 proteins (GA receptors) elevate their direct interaction with DELLA proteins in the nucleus, trigger DELLA protein's ubiquitination and proteasome-mediated degradation, and thus release PIF3 from the negative effect of DELLA proteins.

Light and GA interact during Arabidopsis thaliana seedling development, regulating hypocotyl elongation, cotyledon opening and light-responsive gene expression; their pathways seem to converge at regulation of the abundance of DELLA proteins (GA pathway repressors $)^{3,6}$. Arabidopsis has five DELLA proteins-RGA, GAI, RGL1, RGL2 and RGL3 - defined by their unique DELLA domain and a conserved GRAS domain ${ }^{4}$. To analyse them in vivo, we raised antibodies against endogenous RGA and generated transgenic Arabidopsis expressing each of the five DELLA proteins with tandem affinity purification (TAP) tags (Supplementary Fig. 1). The response of DELLA protein levels to exogenously applied $\mathrm{GA}_{3}$ (an active form of GA) or PAC (paclobutrazol, a GA biosynthesis inhibitor) was examined. We found that one-hour-long GA treatment eliminates the majority of DELLA proteins, and this GA effect can be largely prevented by $100 \mu \mathrm{M}$ MG132 (a 26S proteasome-specific inhibitor). PAC, on the other hand, promotes over-accumulation of DELLA proteins (Fig. 1). These results show for the first time in Arabidopsis that all the DELLA proteins are under negative control by GA and the proteasome. Next, we generated lines expressing TAPtagged RGA $\Delta 17$ and GAI $\Delta 17$, which lack a 17 amino acid motif within the DELLA domain that is required for GA-induced degradation $^{7,8}$. As expected, TAP-RGA $\Delta 17$ and TAP-GAI $\Delta 17$ are completely resistant to GA and accumulate at higher levels than wild-type proteins, which cannot be further increased by PAC (Fig. 1, and

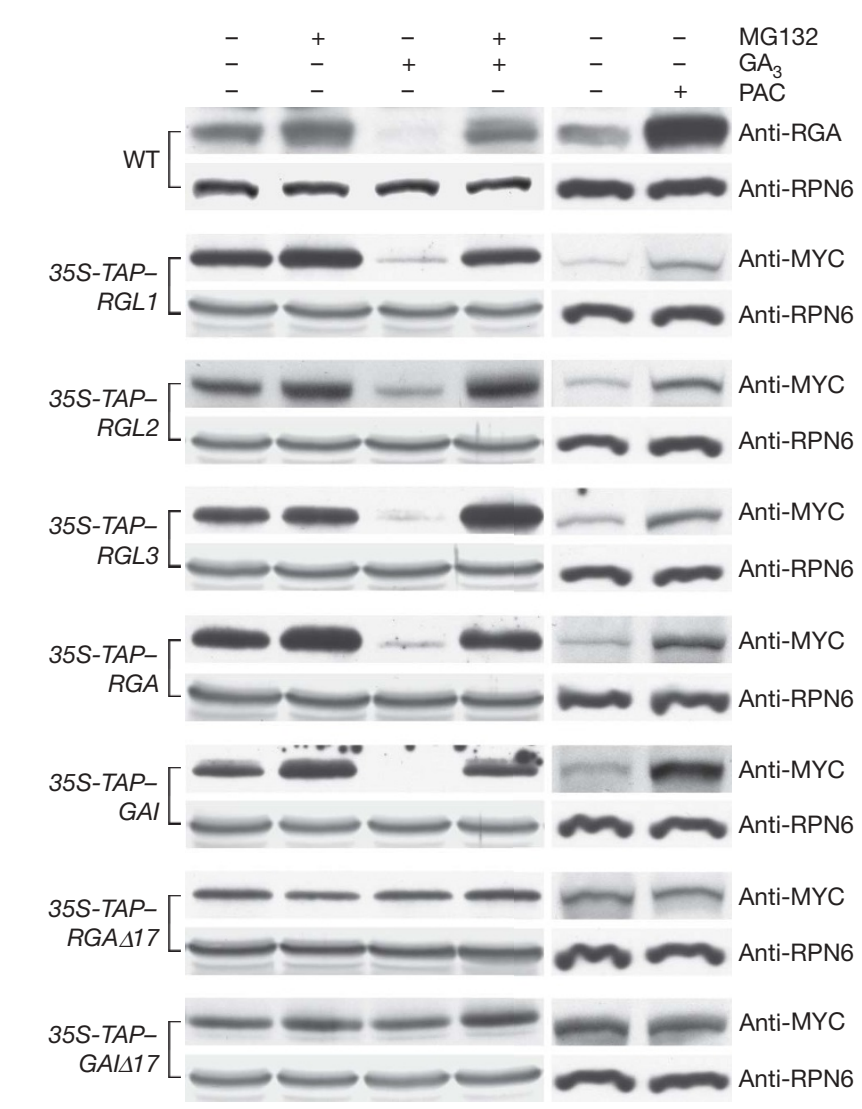

Figure 1 | Effect of $\mathrm{GA}_{3}, \mathrm{MG} 132$ and PAC on DELLA protein abundance. Immunoblot analysis of RGA (by anti-RGA antibody) and TAP-DELLA proteins (by anti-MYC antibody) in various light-grown Arabidopsis seedlings (genotypes labelled to the left of each panel) treated with different combinations of $\mathrm{GA}_{3}, \mathrm{MG} 132$ and PAC. Panels on the left (four lanes) and panels on the right (two lanes) are from two independent experiments using different protein gel systems. RPN6 immunoblotting (by anti-RPN6 antibody) is used as a loading control. WT, wild type.

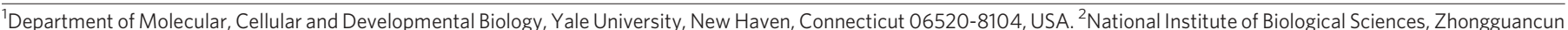

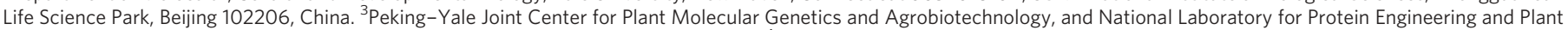

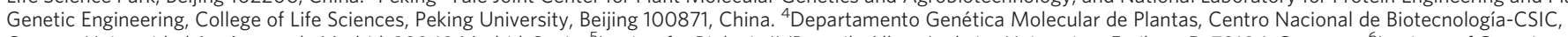

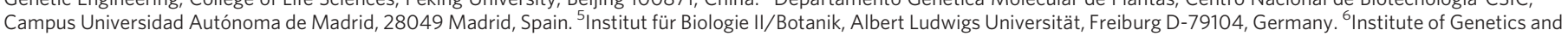

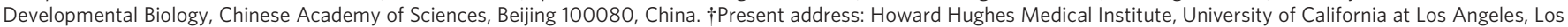
Angeles, California 90095-1606, USA.

*These authors contributed equally to this work. 
Supplementary Fig. 1b). Arabidopsis plants that overexpress these proteins show a dominant dwarf phenotype, reflecting enhanced DELLA activity (Supplementary Fig. 2), which also suggests that TAP-DELLA proteins retain normal DELLA function.

Inhibition of hypocotyl elongation, an important characteristic of photomorphogenesis, is shown to be repressed by GA in the dark and
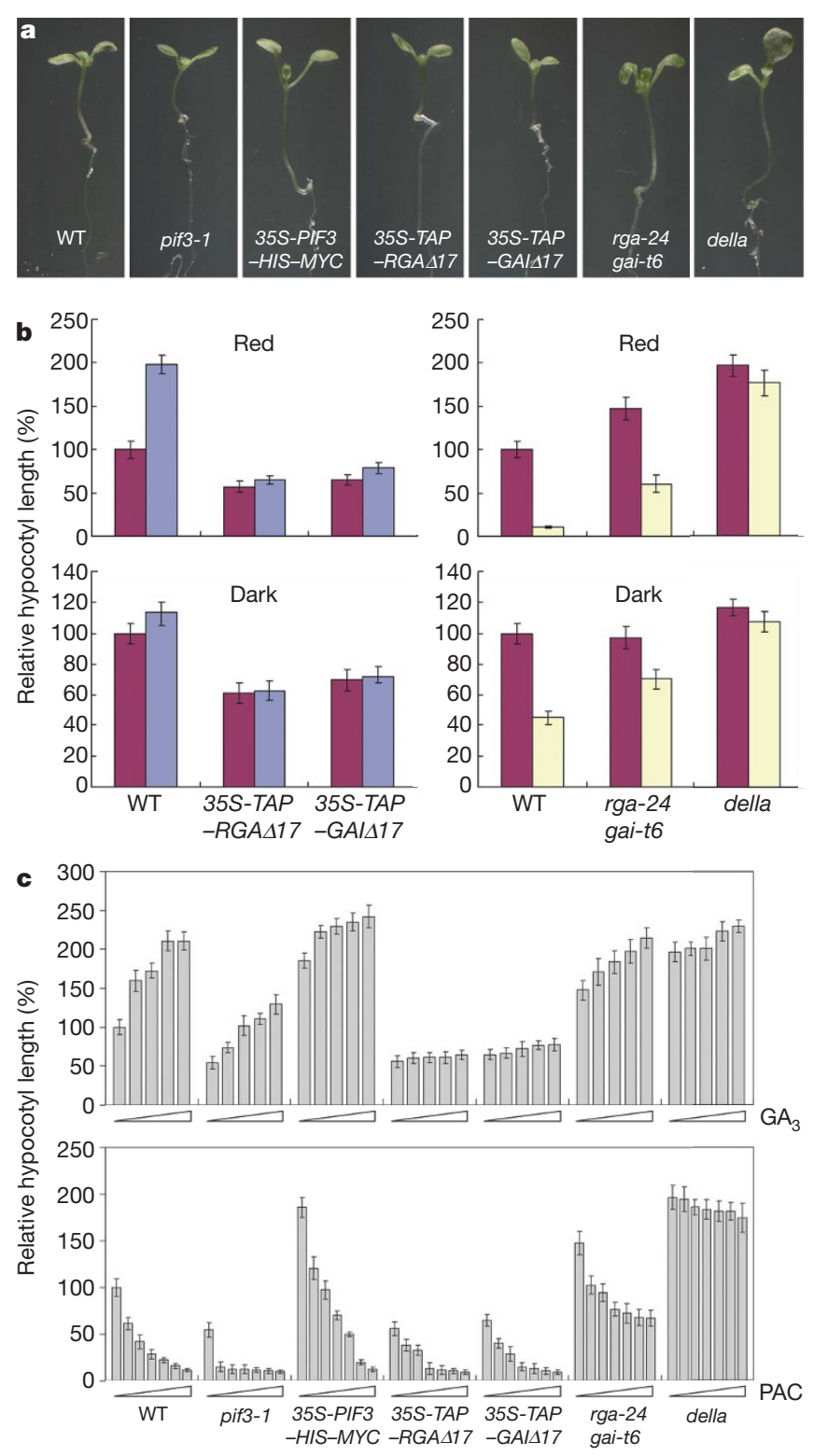

d

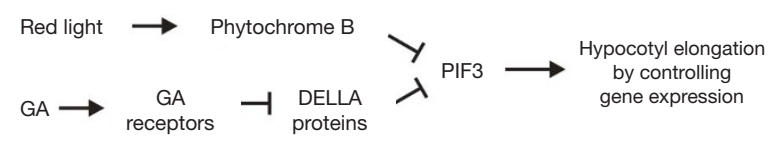

Figure 2 | DELLA proteins and PIF3 have opposite roles in regulating Arabidopsis hypocotyl elongation. a, Images of red-light-grown seedlings. b, Hypocotyl length measurement (mean \pm s.d.) of untreated seedlings (red), or seedlings treated with $10 \mu \mathrm{M} \mathrm{GA}_{3}$ (blue) or $1 \mu \mathrm{M}$ PAC (yellow).

c, Hypocotyl length measurement (mean \pm s.d.) of red-light-grown seedlings treated with increasing amounts of $\mathrm{GA}_{3}$ or PAC (see Methods). The concentrations of $\mathrm{GA}_{3}$ used are $0,0.5 \mu \mathrm{M}, 1 \mu \mathrm{M}, 2 \mu \mathrm{M}$ and $5 \mu \mathrm{M}$ (from left to right). The concentrations of PAC were $0,0.01 \mu \mathrm{M}, 0.02 \mu \mathrm{M}, 0.05 \mu \mathrm{M}, 0.1 \mu \mathrm{M}$, $0.2 \mu \mathrm{M}$ and $0.5 \mu \mathrm{M}$ (from left to right). In $\mathbf{b}$ and $\mathbf{c}$, hypocotyl length of untreated wild-type seedlings is set to $100 \%$. d, Simplified diagram depicting the genetic interaction of light and GA in the control of hypocotyl elongation by PIF3 and DELLA proteins. della, rga-t2 gai-t6 rgl1-1 rgl2-1 rgl3-1. promoted by DELLA proteins in the light ${ }^{3,6}$. We further examined the possible mechanism of DELLA proteins in regulating photomorphogenesis. Arabidopsis seedlings have longer hypocotyls on GA-containing medium, whereas PAC dramatically inhibits the elongation of hypocotyls (Fig. 2a, b). Furthermore, the GA effect is more drastic in red light than in dark (Fig. 2b), consistent with the notion that the endogenous GA level is higher in dark-grown seedlings. In addition, 35S-TAP-RGA 17 and 35S-TAP-GAIA 17 plants have much shorter hypocotyls than wild type, which cannot be rescued by GA. On the contrary, the hypocotyl of rga-24 gai-t6 double mutants is longer than that of wild type, and is only partially inhibited by PAC. In a pentuple mutant (della) of all five DELLA genes, the hypocotyl length is comparable to that of GA-treated wild type, and PAC has no significant effect (Fig. 2a, b). Therefore, we reasoned that GA controls hypocotyl growth and affects photomorphogenesis status, mainly by regulating DELLA protein abundance.

DELLA proteins are proposed to be transcription factors ${ }^{4}$, and are required to localize to the nucleus for their function ${ }^{9,10}$. Genomic studies have revealed a number of GA-responsive genes that are regulated by DELLA genes ${ }^{5}$. However, using the chromatin immunoprecipitation (ChIP) technique in 35S-TAP-DELLA lines, we were unable to observe specific binding of DELLA proteins to any of the 38 GA-responsive gene promoters tested (Supplementary Table 1). Thus, we hypothesize that DELLA proteins might regulate gene expression indirectly by controlling transcription factors. Because light and DELLA proteins both regulate hypocotyl growth, it seems possible that one, or more, of the well-known photomorphogenesisrelated transcription factors might be a target of DELLA proteins. Among them, PIF3 is a good candidate, because it promotes hypocotyl elongation in red light ${ }^{11}$ - the opposite of DELLA's function (Fig. 2a). Moreover, PIF3 has DNA-binding activity ${ }^{12}$, interacts with the active form of phytochrome $\mathrm{B}$ (phyB) $)^{13,14}$, and is negatively regulated by phytochrome through the ubiquitin/proteasome pathway ${ }^{15-17}$, indicating it mediates signalling between light and gene expression. We observed that the pif3-1 mutant has a short hypocotyl, and is partially resistant to GA and hypersensitive to PAC, mimicking 35S-TAP-RGA 17 and 35S-TAP-GAIA17 plants, whereas the PIF3 overexpression line shows a long hypocotyl and is hyposensitive to PAC, in a similar manner to GA-treated plants and rga-24 gai-t6 and della mutants (Fig. 2c). These results imply that DELLA proteins may negatively regulate PIF3 in the control of hypocotyl elongation, representing a convergent point of light and GA pathways (Fig. 2d).

This regulation is probably mediated through physical interaction between PIF3 and DELLA proteins, as suggested by yeast two-hybrid and in vitro pull-down assays (Fig. 3a, d, and Supplementary Fig. 3). Moreover, bimolecular fluorescence complementation (BiFC) analysis detects direct RGA-PIF3 interaction in the nuclei of living plant cells (Fig. 3b). We further investigated this interaction using an immunoprecipitation approach. As shown in Fig. 3c, interaction between RGA and PIF3 is observed in dark-grown seedlings, in which PIF3 protein accumulates to reasonable abundance ${ }^{15-17}$. The interaction is also detectable in red light, when light-induced proteasomal degradation of PIF3 (refs 15-17) is blocked. The interaction is dependent on RGA abundance, such that PAC increases RGA-PIF3 interaction, whereas GA abolishes RGA accumulation and thus PIF3 is released. Importantly, under the condition that RGA-PIF3 interaction is enhanced, PIF3's effect on hypocotyl growth is largely impaired, and vice versa (Figs 2c, 3c), indicating that RGA-bound PIF3 has reduced activity. We tested whether DELLA proteins influence the previously reported interaction between phytochrome and PIF3 (refs 13, 14) by analysing the formation of nuclear speckles containing both phyB and PIF3 (ref. 15). Evidently, phyB-PIF3 interaction is essentially not affected by altering DELLA protein abundance (Supplementary Fig. 4). Therefore, DELLA protein binding most probably affects PIF3's transcription-regulation activity towards its target genes. 
This notion is supported by the observation that the RGA-PIF3 interaction in vitro is specifically inhibited by pre-incubating PIF3 with its cognate binding site, a G-box-containing DNA probe ${ }^{12}$
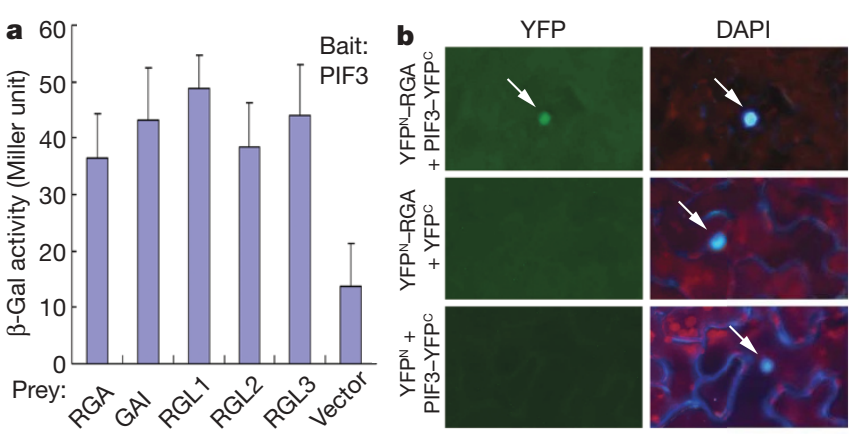

c

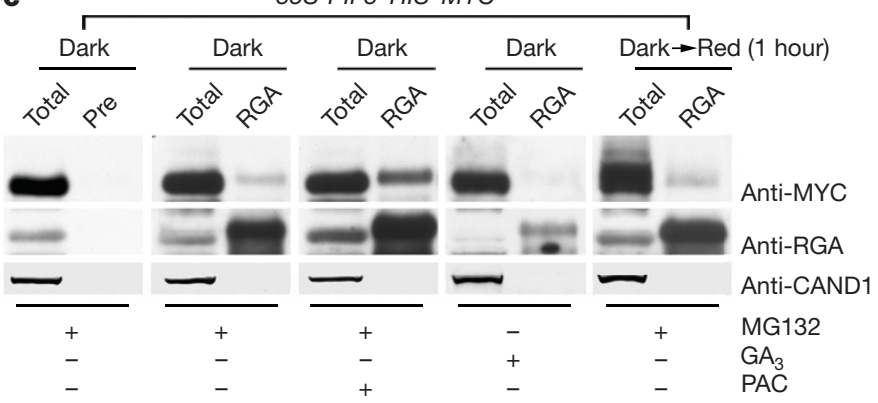

d
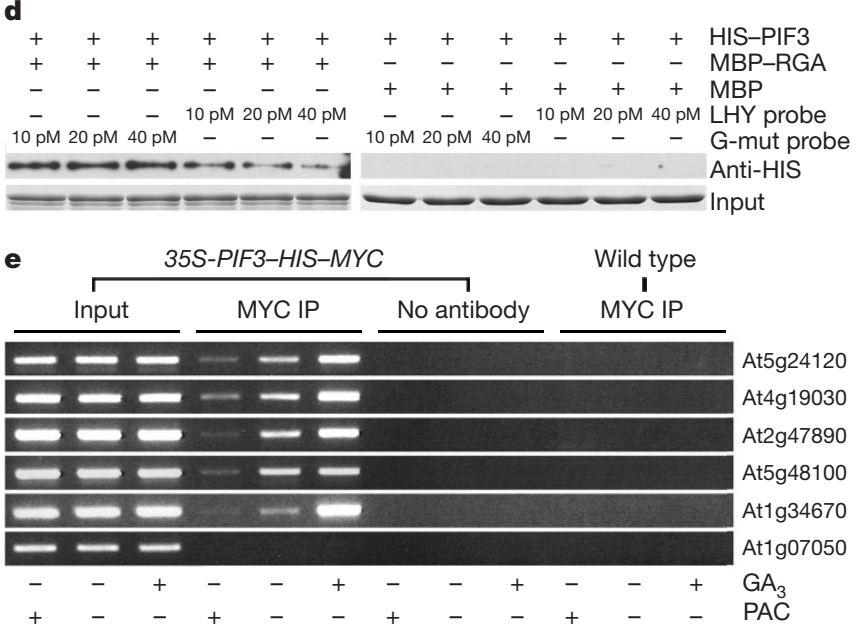

f

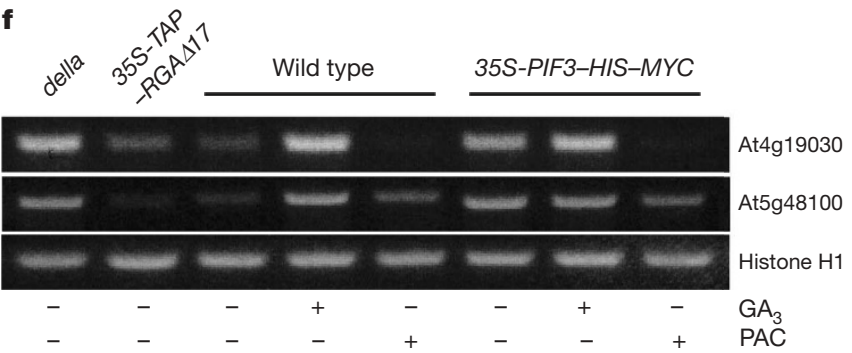

Figure 3 | DELLA proteins bind PIF3 and inhibit PIF3 activity towards its target genes. a, $\beta$-galactosidase activities from yeast two-hybrid assays (mean \pm s.d.). b, BiFC analysis of RGA and PIF3. The positions of nuclei are indicated by arrows. c, Co-immunoprecipitation of RGA with PIF3 in 35SPIF3-His-MYC seedlings. 'RGA' and 'Pre' indicate immunoprecipitation by anti-RGA antibody and pre-immune sera, respectively. d, Pull-down assays between His-PIF3 and MBP-RGA. The precipitated His-PIF3 was detected by anti-His antibody. MBP-RGA and MBP inputs were stained by Coomassie blue. e, ChIP-PCR analyses in dark-grown seedlings. f, Semiquantitative RT-PCR analyses in dark-grown seedlings. Total, total protein extracts; IP, immunoprecipitation.
(LHY; Fig. 3d, and Supplementary Fig. 3), which provides evidence that RGA-PIF3 and PIF3-DNA bindings are antagonistic. To test this in vivo, we selected five putative PIF3 target genes by analysing the published literature as well as taking into account results we obtained from a ChIP microarray analysis focused on PIF3, using a recently reported method ${ }^{18}$. By ChIP-PCR, we confirmed that these five promoters are bound by PIF3 as expected. In addition, we found that when DELLA protein level is increased by PAC, PIF3-promoter binding is severely reduced. On the other hand, removing DELLA proteins by GA treatment generally leads to enhanced occupancy of PIF3 on the promoters (Fig. 3e). We also noticed that, whereas GA and PAC do not significantly affect nuclear PIF3-MYC protein levels, they have slightly opposite effects on PIF3-MYC immunoprecipitation (Supplementary Fig. 5), which might be due to higher affinity of MYC antibody towards free PIF3-MYC than RGA-bound PIF3MYC. Among the PIF3 target genes, At5g2120, At4g19030, At2g47890 and At1g34670 show light-responsive expression ${ }^{19}$. Interestingly, differential expression of At5g24120, At4g19030 and At2g47890 have also been reported in genomic studies focused on gene expression regulation by GA, PIF3 or DELLA genes ${ }^{5,20,21}$. Subsequently, we used PCR with reverse transcription (RT-PCR) to check whether PIF3-promoter binding indeed affects gene expression. As shown in Fig. 3f, overexpressing PIF3 and reducing DELLA protein abundance (della mutant or GA treatment) have similar effect on the expression of two representative PIF3 target genes, whereas increasing DELLA protein abundance (overexpressing RGA $\Delta 17$ or PAC treatment) has the opposite effect. Overall, we demonstrate that DELLA proteins antagonize PIF3 function by protein-protein interaction and sequestration, which at least partly explains their effect on gene expression and the coordinated control of hypocotyl growth by light and GA.

We next examined how the GA signal is relayed to affect DELLA protein abundance and thus DELLA-PIF3 interaction. Recently, GID1 proteins have been shown to act as nuclear GA receptors ${ }^{22-25}$. Through isolating and analysing Arabidopsis gidl mutants, we obtained results that are consistent with those reports ${ }^{24,25}$, suggesting that GID1s are required for normal GA signalling and participate in light-induced development, possibly by inducing DELLA protein degradation (Supplementary Note 1 and Supplementary Fig. 6). We also confirmed the reported GA-dependent GID1-DELLA interaction $^{22-25}$, which requires the 17 amino acid motif within the DELLA domain, in yeast two-hybrid assays (Fig. 4a, b, and Supplementary Fig. 7). In addition, BiFC analysis of GID1c and RGA demonstrates their direct interaction in the nuclei of living plant cells (Fig. 4c). To test the effect of GA on GID1-DELLA interaction in planta, we used transgenic Arabidopsis expressing each of the three GID1 proteins with YFP or epitope tags for immunoprecipitation analyses. As shown in Fig. 4d, interaction of GIDla with each of the five DELLA proteins is detectable, and is greatly enhanced by GA. Furthermore, with MG132 treatment, immunoprecipitated DELLA proteins contain high-molecular-weight protein species, which react specifically with anti-ubiquitin antibody. Increasing amounts of these DELLA proteins that are predicted to be multi-ubiquitinated can be detected after GA treatment (Fig. 4d, e). On the other hand, GAI $\Delta 17$ shows no detectable interaction with GID1a and a nearly complete loss of multi-ubiquitination (Fig. 4d), suggesting that interaction with GID1s is essential for the multi-ubiquitination of DELLA proteins. We extended the interaction study to all three GID1 proteins, and the co-immunoprecipitation of each GID1 with endogenous RGA was detected (Fig. $4 \mathrm{f}-\mathrm{h}$ ). In these experiments, we lowered MG132 concentration $(40 \mu \mathrm{M})$ and lengthened GA treatment time (two hours) to only partially inhibit the proteasome and allow a significant portion of RGA to be degraded. Nevertheless, GA-treated seedlings still have more GID1-bound RGA (Fig. 4f-h), implying that GID1-DELLA may be recognized by the ubiquitin/proteasome system as a heteromeric complex, and somehow DELLA proteins are preferentially ubiquitinated and degraded. This is supported by the 
observation that GID1a enhances RGA-SLY1 (E3 ubiquitin ligase subunit) interaction in the presence of $\mathrm{GA}^{24}$. Consistent with previous results in yeast ${ }^{22}$, GID1b binds more RGA in untreated seedlings, and even with PAC treatment a small amount of GID1bbound RGA can be detected (Fig. 4f-h). This implies a possible GAindependent pathway for GID1b to target DELLA proteins, which might be critical to keep DELLA proteins in check when the GA level is low.

The results reported here support a conclusion that GA signalling is initiated when GA molecules, the biosynthesis of which is induced by light ${ }^{3}$, are sensed and bound by nuclear GID1 proteins. Then, GAcharged GID1s interact with DELLA proteins in the nucleus and target them for proteasomal degradation. When DELLA protein abundance is reduced, their interactive partners, for example, light-responsive and phytochrome-interacting transcription factors such as PIF3, are released from sequestration, and bind to their target promoters and regulate gene expression (Fig. 4i). PIF3 belongs to a transcription factor family defined by a conserved bHLH (basichelix-loop-helix) domain, which has implicated function in DNA binding and dimerization ${ }^{26}$. In a similar way to PIF3, PIF4 (another phytochrome-interacting bHLH transcription factor) is also negatively regulated by DELLA proteins ${ }^{27}$. Moreover, DELLA proteins are shown to interact with the DNA-binding bHLH domain ${ }^{27}$, consistent with our observation that RGA-PIF3 and PIF3-promoter interactions are mutually exclusive (Fig. 3). Two other bHLH proteins, PIL5 and SPT, are also involved in light and GA signalling, and have PIF3like roles in hypocotyl growth ${ }^{28,29}$, making them potential targets of DELLA proteins as well. Collectively, it is highly plausible that,
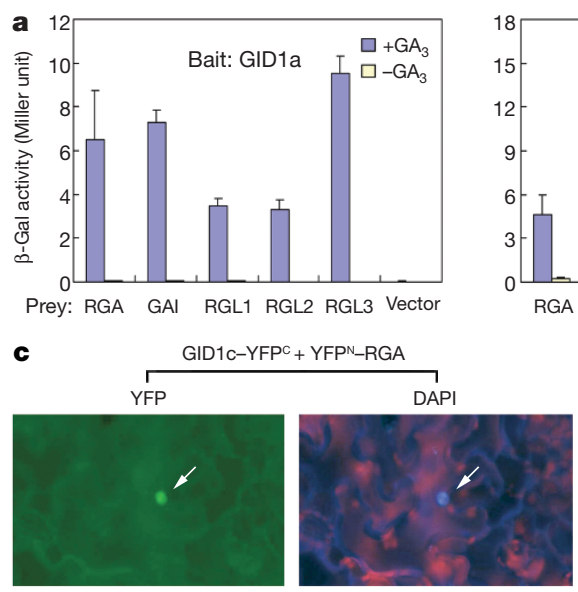

d
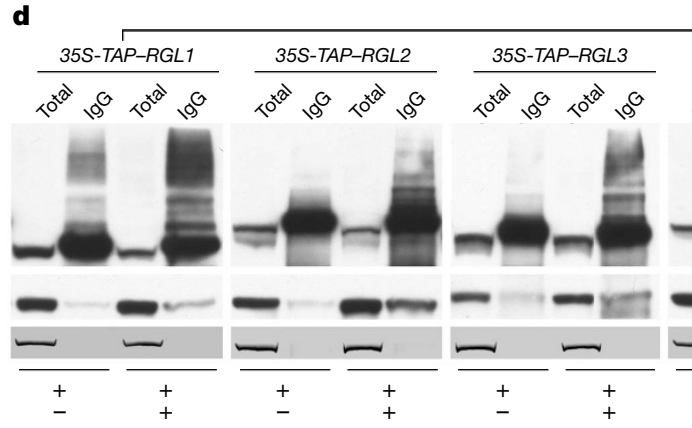

35S-GID1a-YFP +
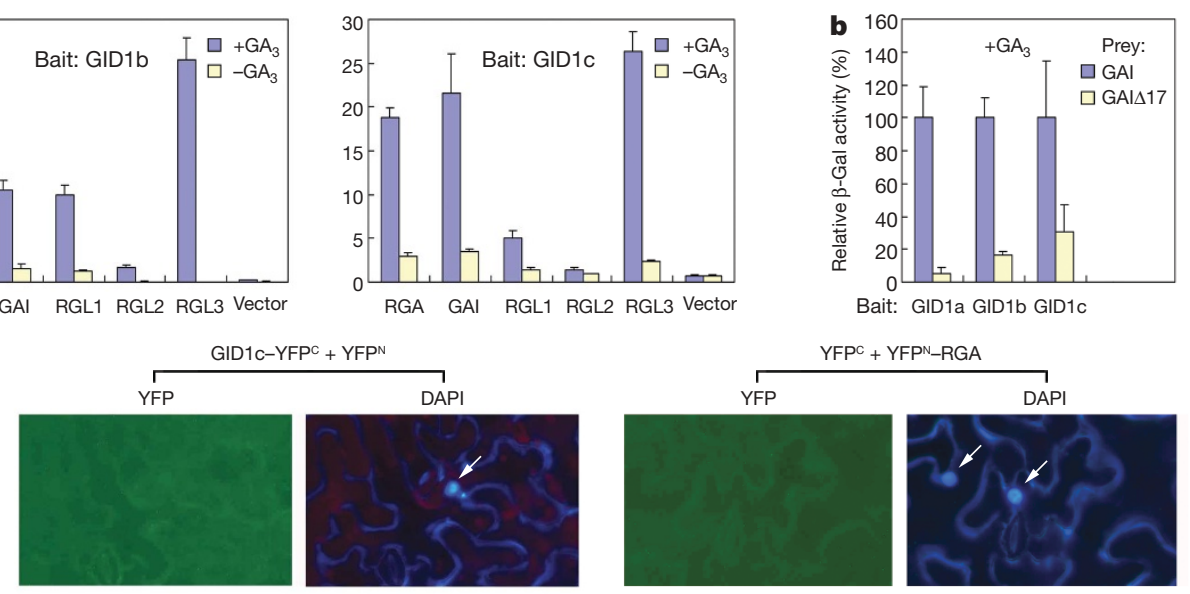

Bait: GID1a GID1b GID1c
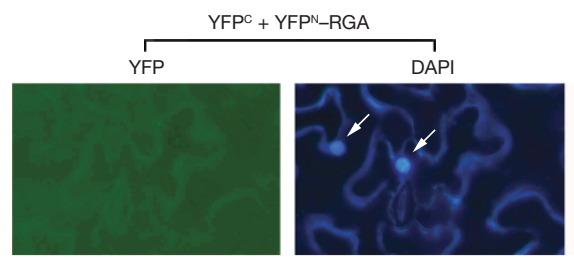

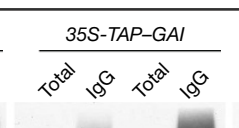

< e

35S-TAP-RGA

10

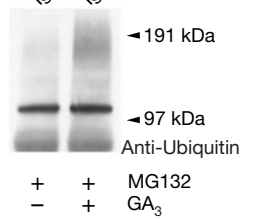

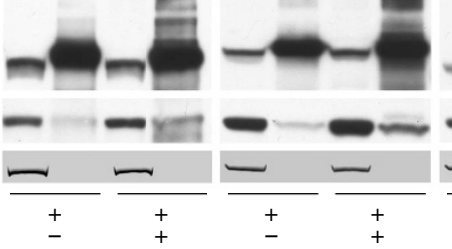
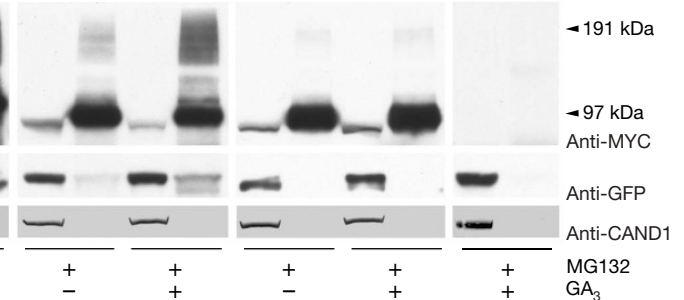

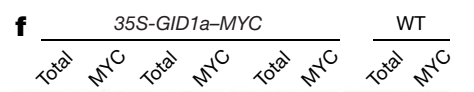

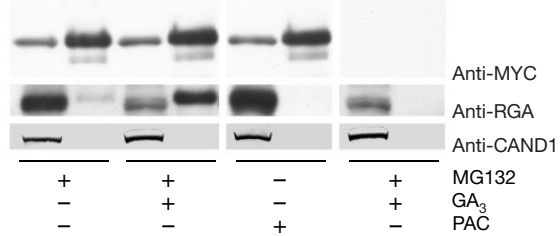

g 35S-GID1b-Flag

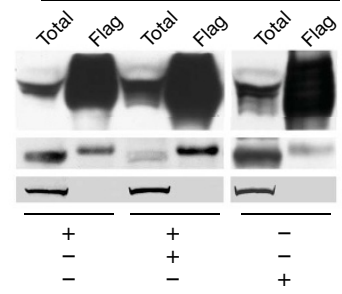

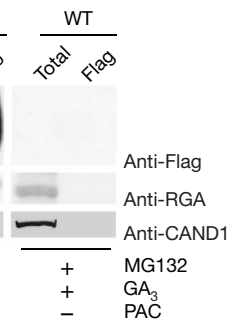

h $35 S-G I D 1 C-H A$

$\frac{W T}{20^{20}+x^{2}}$
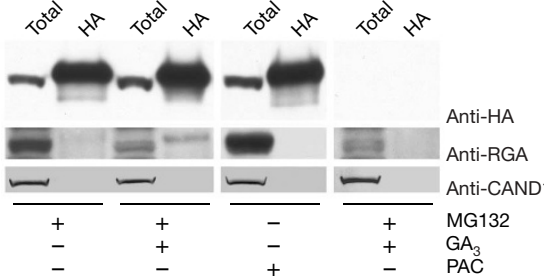

i

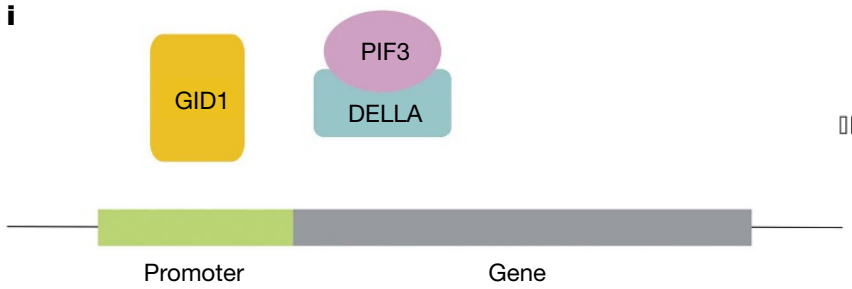

Figure 4 | GA-dependent interaction between GID1s and DELLA proteins. a, b, $\beta$-galactosidase activities from yeast two-hybrid assays (mean \pm s.d.). In b, $\beta$-galactosidase activities from GAI-GID1 interactions are set to $100 \%$. c, BiFC analysis of GID1c and RGA. The positions of nuclei are indicated by arrows. d, TAP-DELLA proteins interact with GID1a-YFP. e, Detection of

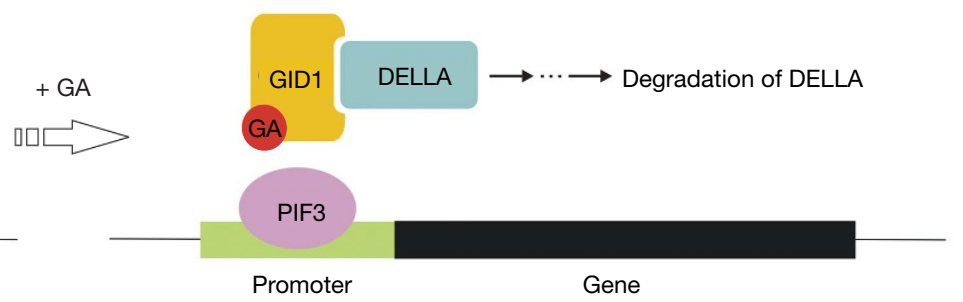

multi-ubiquitinated TAP-RGA. In d, e, 'IgG' indicates immunoprecipitation by IgG-conjugated beads. $\mathbf{f}-\mathbf{h}, \mathrm{RGA}$ interacts with GID1a-MYC

(f), GID1b-Flag (g), and GID1c-HA(haemagglutinin) (h). 'MYC', 'Flag' and 'HA' indicate immunoprecipitation by respective antibodies. i, A working model of the nuclear protein interaction cascade in GA signalling. 
through modulating multiple phytochrome-interacting transcription factors, DELLA proteins play a key part in integrating the regulatory effect of light and GA on gene expression and plant development.

\section{METHODS SUMMARY}

The procedures for Arabidopsis plant growth, yeast two-hybrid analyses, protein and chromatin immunoprecipitations, and subcellular localization studies are described previously $y^{15,18,30}$. CAND1 is used as a negative control in protein immunoprecipitation experiments. Histone $\mathrm{H} 1$ is used as an internal control in RT-PCR. Primers used in ChIP-PCR and RT-PCR are listed in Supplementary Tables 1 and 2. MG132 treatment is carried out by vacuum infiltration. GA and PAC treatments are carried out by either applying $\mathrm{GA}_{3}$ to the seedlings or supplementing plant growth medium with $\mathrm{GA}_{3}$ or PAC. BiFC experiments are carried out between transiently expressed Arabidopsis proteins in tobacco leaves. In vitro pull-down assays are performed using recombinant proteins purified from bacteria, in the presence of either a canonical G-box containing DNA probe (LHY) or a mutant G-box-containing DNA probe (G-mut).

Full Methods and any associated references are available in the online version of the paper at www.nature.com/nature.

\section{Received 28 September; accepted 1 November 2007.}

1. Fleet, C. M. \& Sun, T. P. A. DELLAcate balance: the role of gibberellin in plant morphogenesis. Curr. Opin. Plant Biol. 8, 77-85 (2005).

2. Itoh, H., Matsuoka, M. \& Steber, C. M. A role for the ubiquitin-26S-proteasome pathway in gibberellin signaling. Trends Plant Sci. 8, 492-497 (2003).

3. Achard, P. et al. DELLAs contribute to plant photomorphogenesis. Plant Physiol. 143, 1163-1172 (2007).

4. Bolle, C. The role of GRAS proteins in plant signal transduction and development. Planta 218, 683-692 (2004).

5. Cao, D., Cheng, H., Wu, W., Soo, H. M. \& Peng, J. Gibberellin mobilizes distinct DELLA-dependent transcriptomes to regulate seed germination and floral development in Arabidopsis. Plant Physiol. 142, 509-525 (2006).

6. Alabadi, D., Gil, J., Blazquez, M. A. \& Garcia-Martinez, J. L. Gibberellins repress photomorphogenesis in darkness. Plant Physiol. 134, 1050-1057 (2004).

7. Dill, A., Thomas, S. G., Hu, J., Steber, C. M. \& Sun, T. P. The Arabidopsis F-box protein SLEEPY1 targets gibberellin signaling repressors for gibberellin-induced degradation. Plant Cell 16, 1392-1405 (2004).

8. $\mathrm{Fu}, \mathrm{X}$. et al. The Arabidopsis mutant sleepy $7^{\mathrm{gar} 2-1}$ protein promotes plant growth by increasing the affinity of the $\mathrm{SCF}^{\mathrm{SLY} 1} \mathrm{E} 3$ ubiquitin ligase for DELLA protein substrates. Plant Cell 16, 1406-1418 (2004).

9. Silverstone, A. L. et al. Repressing a repressor: gibberellin-induced rapid reduction of the RGA protein in Arabidopsis. Plant Cell 13, 1555-1566 (2001)

10. Itoh, H., Ueguchi-Tanaka, M., Sato, U., Ashikari, M. \& Matsuoka, M. The gibberellin signaling pathway is regulated by the appearance and disappearance of SLENDER RICE1 in nuclei. Plant Cell 14, 57-70 (2002).

11. $\mathrm{Kim}$, J. et al. Functional characterization of phytochrome interacting factor 3 in phytochrome-mediated light signal transduction. Plant Cell 15, 2399-2407 (2003).

12. Martinez-Garcia, J. F., Huq, E. \& Quail, P. H. Direct targeting of light signals to a promoter element-bound transcription factor. Science 288, 859-863 (2000).

13. Ni, M., Tepperman, J. M. \& Quail, P. H. Binding of phytochrome B to its nuclear signalling partner PIF3 is reversibly induced by light. Nature 400, 781-784 (1999).

14. Zhu, Y., Tepperman, J. M., Fairchild, C. D. \& Quail, P. H. Phytochrome B binds with greater apparent affinity than phytochrome A to the basic helix-loop-helix factor PIF3 in a reaction requiring the PAS domain of PIF3. Proc. Natl Acad. Sci. USA 97, 13419-13424 (2000).

15. Bauer, D. et al. Constitutive photomorphogenesis 1 and multiple photoreceptors control degradation of phytochrome interacting factor 3 , a transcription factor required for light signaling in Arabidopsis. Plant Cell 16, 1433-1445 (2004).

16. Park, E. et al. Degradation of phytochrome interacting factor 3 in phytochromemediated light signaling. Plant Cell Physiol. 45, 968-975 (2004).
17. Al-Sady, B., Ni, W., Kircher, S., Schafer, E. \& Quail, P. H. Photoactivated phytochrome induces rapid PIF3 phosphorylation prior to proteasome-mediated degradation. Mol. Cell 23, 439-446 (2006).

18. Lee, J. et al. Analysis of transcription factor HY5 genomic binding sites revealed its hierarchical role in light regulation of development. Plant Cell 19, 731-749 (2007).

19. Jiao, Y., Ma, L., Strickland, E. \& Deng, X. W. Conservation and divergence of lightregulated genome expression patterns during seedling development in rice and Arabidopsis. Plant Cell 17, 3239-3256 (2005).

20. Monte, E. et al. The phytochrome-interacting transcription factor, PIF3, acts early, selectively, and positively in light-induced chloroplast development. Proc. Natl Acad. Sci. USA 101, 16091-16098 (2004).

21. Ogawa, M. et al. Gibberellin biosynthesis and response during Arabidopsis seed germination. Plant Cell 15, 1591-1604 (2003).

22. Ueguchi-Tanaka, M. et al. GIBBERELLIN INSENSITIVE DWARF1 encodes a soluble receptor for gibberellin. Nature 437, 693-698 (2005).

23. Nakajima, M. et al. Identification and characterization of Arabidopsis gibberellin receptors. Plant J. 46, 880-889 (2006).

24. Griffiths, J. et al. Genetic characterization and functional analysis of the GID1 gibberellin receptors in Arabidopsis. Plant Cell 18, 3399-3414 (2007).

25. Willige, B. C. et al. The DELLA domain of GA INSENSITIVE mediates the interaction with the GA INSENSITIVE DWARF1A gibberellin receptor of Arabidopsis. Plant Cell 19, 1209-1220 (2007).

26. Toledo-Ortiz, G., Huq, E. \& Quail, P. H. The Arabidopsis basic/helix-loop-helix transcription factor family. Plant Cell 15, 1749-1770 (2003).

27. de Lucas, M. et al. A molecular framework for light and gibberellin control of cell elongation. Nature doi:10.1038/nature06520.

28. Oh, E. et al. PIL5, a phytochrome-interacting basic helix-loop-helix protein, is a key negative regulator of seed germination in Arabidopsis thaliana. Plant Cell 16, 3045-3058 (2004).

29. Penfield, S. et al. Cold and light control seed germination through the bHLH transcription factor SPATULA. Curr. Biol. 15, 1998-2006 (2005).

30. Feng, S. et al. Arabidopsis CAND1, an unmodified CUL1-interacting protein, is involved in multiple developmental pathways controlled by ubiquitin/ proteasome-mediated protein degradation. Plant Cell 16, 1870-1882 (2004).

Supplementary Information is linked to the online version of the paper at www.nature.com/nature.

Acknowledgements We thank J. Lee, K. He and I. Lee for providing unpublished results of their ChIP-microarray studies; J. A. Sullivan for MYC- and HA-tag vectors; F. Nagy for the YFP-tag vector; X. P. Wang for the Flag-tag vector; P.H. Quail for the pPIF3-RSETb plasmid; G. Choi for pif3-1 and 35S-PIF3-His-MYC seeds; T. P. Sun for rga-24 and rg/2-13 seeds; N. P. Harberd for gai-t6 seeds;

S. P. Dinesh-Kumar for tobacco seeds; and F. Parcy for providing the BiFC vector system. This work was supported by a grant from the National Institutes of Health to X.W.D., by a grant from the Ministry of Science and Technology of China to National Institute of Biological Sciences at Beijing, by a 985 program fund from Peking University and the Ministry of Education of China to the Peking-Yale Joint Center laboratory, and by a grant from Program for New Century Excellent Talents in Peking University to L.-M.F. C.M. is a recipient of a postdoctoral fellowship from the Spanish Ministerio de Educacion y Ciencia. J.M.I.-P. is a recipient of a predoctoral fellowship from the Spanish Ministerio de Educacion y Ciencia. E.S. and S.K. were supported by grants from the Deutsche Forschungsgesellschaft.

Author Contributions X.W.D. conceived the project, and S.F. and X.W.D. together designed the experiments. L.-M.F. designed some of the experiments. S.F. and G.G. performed chromatin immunoprecipitation. C.M. and G.G. analysed the gid1 mutants and performed RT-PCR. Y.W. made the anti-RGA antibody. Y.W., L.C., F.W. and L.Y. performed the yeast two-hybrid analyses. J.Z. and F.W. performed in vitro pull-down assays. C.M. and J.M.I.-P. performed the BiFC experiments. S.K. and E.S. performed phyB and PIF3 subcellular localization studies. X.F. provided the rga-24 gai-t6 double and della pentuple mutants. S.F. performed all other experiments. S.F. and X.W.D. wrote the manuscript.

Author Information Reprints and permissions information is available at www.nature.com/reprints. Correspondence and requests for materials should be addressed to X.W.D. (xingwang.deng@yale.edu). 


\section{METHODS}

Plant materials and growth conditions. The wild-type Arabidopsis ecotypes used in this study are Landsberg erecta and Columbia-0. The T-DNA insertion alleles, gid1a-2 and gid1c-1, were verified by PCR-based genotyping. They turn out to be identical to the alleles published in a recent report ${ }^{24}$. The point mutation alleles, gid1b-2 and gid1b-3, were isolated from the Arabidopsis TILLING collection (http://tilling.fhcrc.org/). To grow gidla-2 gid1b-3 gid1c-1 triple mutant, embryos are mechanically removed from seed coat and allowed to germinate on MS medium plates. For hypocotyl elongation, ChIP-PCR, RTPCR and subcellular localization experiments, Arabidopsis seeds are sown on filter papers in MS medium plates and placed under continuous white light. After $24 \mathrm{~h}$, filter papers with seeds are transferred onto new MS plates, or MS plates containing various concentrations of $\mathrm{GA}_{3}$ or PAC, and then placed in the dark or red light for six days.

Generation of transgenic Arabidopsis lines. DNA fragments containing fulllength open reading frames of five DELLA genes (RGA, GAI, RGL1, RGL2 and $R G L 3$ ) and two deletion mutants (RGA $\triangle 17$ and GAIA 17) are cloned via Gateway reactions into the binary $\mathrm{N}$-terminal TAP-tag vector ${ }^{31}$. DNA fragments containing full-length open reading frames of GID1 genes (GID1a, GID1b, and GID1c) are cloned into YFP-, Flag-, MYC- and HA-tag vectors, respectively. Subsequently, the fragments that contain the gene-coding sequence plus the tag are subcloned into the binary pJIM19 vector ${ }^{18}$. The binary constructs are introduced into Arabidopsis by Agrobacterium-mediated transformation.

GA, MG132 and PAC treatments. For protein immunoprecipitation and immunoblotting experiments, 10-day-old seedlings are vacuum-infiltrated with $40 \mu \mathrm{M}$ or $100 \mu \mathrm{M}$ MG132 (dissolved in DMSO) or DMSO alone for ten minutes and kept immersed in the same solution for two hours. Then $\mathrm{GA}_{3}$ is added in the solution to a final concentration of $100 \mu \mathrm{M}$, and after one- or two-hour incubation, plant tissues are harvested. For PAC treatment, Arabidopsis seeds are sown on MS medium, and after $24 \mathrm{~h}$ they are transferred to MS medium containing $1 \mu \mathrm{M}$ PAC. Ten-day-old seedlings are harvested.

Yeast two-hybrid and immunoprecipitation assays. Yeast two-hybrid analyses and protein immunoprecipitation using antibody- and IgG-conjugated beads are performed as described previously ${ }^{30}$. A complementary DNA fragment corresponding to amino acids 1 to 201 of RGA is cloned into Escherichia coli expression vector pET32a, and purified recombinant protein is used to immunize rabbits for generating polyclonal antibodies. The anti-CAND1 and antiCSN5 antibodies are described previously ${ }^{30,32}$. For immunoprecipitation with anti-RGA antibodies, $10 \mu \mathrm{l}$ affinity-purified anti-RGA antibodies are incubated with protein extracts for four hours. Then, $50 \mu \mathrm{l}$ protein A agarose beads are used to precipitate the immune complex. CAND1 is used as a negative control in protein immunoprecipitation experiments. Chromatin immunoprecipitations
(ChIP) are performed as described previously ${ }^{18}$. For each ChIP, $40 \mu \mathrm{l}$ anti-MYC antibody-conjugated beads are used.

Bimolecular fluorescence complementation analysis. Full-length open reading frames of RGA, PIF3 and GID1c are cloned via Gateway reactions into the binary $\mathrm{pBiFC}$ vectors containing either amino- or carboxy-terminal yellow fluorescence protein (YFP) fragments $\left(\mathrm{YFP}^{\mathrm{N}} \text { and } \mathrm{YFP}^{\mathrm{C}}\right)^{27}$. Each of the three proteins is fused with both YFP fragment vectors, as either an N-terminal or C-terminal fusion, thus resulting in four constructs per protein. For example, we have four fusion configurations for RGA, RGA-YFP ${ }^{\mathrm{C}}, \mathrm{RGA}^{-\mathrm{YFP}^{\mathrm{N}}}, \mathrm{YFP}^{\mathrm{C}}-\mathrm{RGA}$, and $\mathrm{YFP}^{\mathrm{N}}-\mathrm{RGA}$. To test the interactions between RGA and PIF3, and between GID1c and RGA, we examined all eight possible pair-wise combinations via an adapted transient BiFC assay. Leaves of 2-4-week-old tobacco (Nicotiana benthamiana) plants are infiltrated with Agrobacterium (GV3101) strains containing individual BiFC construct pairs, as well as a binary plasmid expressing the p19 protein of tomato bushy stunt virus to suppress gene silencing ${ }^{27}$. Epidermal cell layers are examined under the microscope for fluorescence using the green fluorescent (GFP) filter 3-4 days after infiltration. For RGA and PIF3, we detected positive nuclear BiFC interaction signals in four combinations $\left(\mathrm{YFP}^{\mathrm{N}}-\mathrm{RGA}\right.$ with $\mathrm{YFP}^{\mathrm{C}}-\mathrm{PIF}, \mathrm{YFP}^{\mathrm{N}}-$ RGA with PIF3-YFP ${ }^{\mathrm{C}}$, RGA-YFP ${ }^{\mathrm{N}}$ with YFP YFIF3 and RGA-YFP $^{\mathrm{N}}$ with PIF3$\mathrm{YFP}^{\mathrm{C}}$ ). For GID1c and RGA, we detected positive nuclear BiFC interaction signals in six of the eight pair-wise combinations, except two (YFP ${ }^{\mathrm{C}}-$ GID1c with YFP $^{\mathrm{N}}-$ RGA and YFPC - GID $1 \mathrm{c}$ with RGA-YFP ${ }^{\mathrm{N}}$ ).

In vitro pull-down assays. A DNA fragment encoding full-length RGA protein is cloned into pMal-C2X vector, obtaining a fusion with the maltose-binding protein (MBP). MBP-RGA and MBP proteins are purified from bacteria by amylose resin beads. His-PIF3 protein is purified from bacteria containing pPIF3-RSETb plasmid by Ni-NTA agarose. The canonical G-box containing LHY probe and G-box mutant (G-mut) probe are described previously ${ }^{12}$. Before pull down, His-PIF3 $(1 \mu \mathrm{g})$ is pre-incubated with $10 \mathrm{pM}, 20 \mathrm{pM}$ and $40 \mathrm{pM}$ LHY probe or G-mut probe for $20 \mathrm{~min}$. Then, MBP-RGA $(1 \mu \mathrm{g})$ or MBP $(0.3 \mu \mathrm{g})$ together with amylose resin beads is added and the mixture is incubated for $30 \mathrm{~min}$. After washing five times with buffer supplemented with DNA probes (the same concentrations as used in the pre-incubation), the precipitated PIF3 is analysed by immunoblotting with anti-His antibody.

Subcellular localization study of phyB-YFP and PIF3-CFP(cyan fluorescent protein) fusion proteins. Hypocotyl cells of dark-grown seedlings and darkgrown seedlings exposed to $2 \mathrm{~min}, 1 \mathrm{~h}$ or $18 \mathrm{~h}$ of red-light irradiation are analysed under microscope. Epifluorescent, light and confocal laser scanning microscopy are performed as previously described ${ }^{15}$.

31. Rubio, $\vee$. et al. An alternative tandem affinity purification strategy applied to Arabidopsis protein complex isolation. Plant J. 41, 767-778 (2005).

32. Feng, S. et al. The COP9 signalosome interacts physically with $\mathrm{SCF}^{\mathrm{CO} 11}$ and modulates jasmonate responses. Plant Cell 15, 1083-1094 (2003). 